\title{
Repurposing of FDA-approved Drugs against Active Site and Potential Allosteric Drug Binding Sites of COVID-19 Main Protease
}

\author{
Merve Yuce ${ }^{1}$, Erdem Cicek $^{1}$, Tuğçe İnan ${ }^{1}$, Aslıhan Başak Dağ ${ }^{2}$, Özge Kürkçüoğlu ${ }^{1}$, and \\ Fethiye Aylin Sungur ${ }^{1}$ \\ ${ }^{1}$ Istanbul Technical University \\ ${ }^{2}$ Istanbul Technical University Faculty of Science and Letters
}

April 14, 2021

\begin{abstract}
The novel coronavirus disease 2019 (COVID-19) caused by severe acute respiratory syndrome coronavirus 2 (SARS-CoV-2) still has serious negative effects on health, social life, and economics. Recently, vaccines from various companies have been urgently approved to control SARS-CoV-2 infections. However, any specific antiviral drug has not been confirmed so far for regular treatment. An important target is the main protease (Mpro), which plays a major role in replication of the virus. In this study, Gaussian and residue network models are employed to reveal two distinct potential allosteric sites on Mpro that can be evaluated as drug targets besides the active site. Then, FDA-approved drugs are docked to three distinct sites with flexible docking using AutoDock Vina to identify potential drug candidates. 14 best molecule hits for the active site of Mpro are determined. 6 of these also exhibit high docking scores for the potential allosteric regions. Full-atom molecular dynamics simulations with MM-GBSA method indicate that compounds docked to active and potential allosteric sites form stable interactions with high binding free energy ([?]Gbind) values. [?]Gbind values reach $-52.06 \mathrm{kcal} / \mathrm{mol}$ for the active site, $-51.08 \mathrm{kcal} / \mathrm{mol}$ for the potential allosteric site 1 , and $-42.93 \mathrm{kcal} / \mathrm{mol}$ for the potential allosteric site 2. Energy decomposition calculations per residue elucidate key binding residues stabilizing the ligands that can further serve to design pharmacophores. This systematic and efficient computational analysis successfully determines ivermectine, diosmin and selinexor currently subjected to clinical trials, and further proposes bromocriptine, elbasvir as Mpro inhibitor candidates to be evaluated against SARS-CoV-2 infection
\end{abstract}

\section{Hosted file}

Manuscript.pdf available at https://authorea.com/users/407791/articles/518016-repurposingof-fda-approved-drugs-against-active-site-and-potential-allosteric-drug-binding-sitesof-covid-19-main-protease 